Nahida Sarhan de-Lima ${ }^{1}$ Lídia Roth Matos ${ }^{1}$ Danilo Barreto Matos ${ }^{1}$ Juliana Borges de Lima

Dantas $^{1}$ Vinicius Rabelo Torregrossa ${ }^{1 *}$

\section{Extensive palatal swelling and asymptomatic pulp necrosis in a patient with sickle cell trait: A new case report}

\begin{abstract}
:
Although sickle cell trait (SCT) has been seen as a benign condition over time, data are accumulating of serious complications due to red cell sickling enhanced by hypoxia, acidosis, dehydration, increased blood viscosity, and hypothermia. Asymptomatic pulp necrosis (APN) is a commonly reported oral complication in patients diagnosed with sickle cell disease. However, no data are associating APN in SCT patients. We report a clinical case of an extensive palatal swelling secondary to multiple APN in a patient with SCT. A 37-year-old woman diagnosed with SCT was referred for evaluation of a year-long expanding palatal swelling of the left anterior maxilla. Clinical examination revealed a negative response to the cold test in two intact permanent teeth associated with the lesion, which were referred to endodontic treatment. After that, an exploratory biopsy of the region was performed, which consisted of enucleation and curettage of all the periapical tissue. Histopathological examination confirmed the hypothesis of a periapical granuloma secondary to APN. A degree of clinical suspicion is required by dentists when pulpal necrosis of unknown origin is presented by patients with SCT.
\end{abstract}

Keywords: Sickle cell trait; Asymptomatic pulp necrosis, Periapical granuloma

${ }^{1}$ Faculdade Adventista da Bahia - FADBA, Dental School, Department of Semiology and Clinic, Cachoeira, BA, Brazil.

Correspondence to:

Vinicius Rabelo Torregrossa.

E-mail: viniciusrabelotorregrossa@gmail.com

Article received on June 20, 2021.

Article accepted on September 23, 2021.

DOI: 10.5935/2525-5711.20210017 


\section{INTRODUCTION}

The sickle cell trait is the heterozygous condition of the mutation in the sixth codon of the hemoglobin $\beta$ chain, whereby the glutamic acid is replaced by valine, giving rise to mutated hemoglobin, $\mathrm{HbS}$, which replaces normal hemoglobin, $\mathrm{HbA}^{1}$. Heterozygous individuals with such hemoglobin $\mathrm{S}(\mathrm{HbAS})$ have the sickle cell trait (SCT) and do not present frequent clinical manifestations or demand for medical treatment. This occurs because SCT patients have low percentages of hemoglobin S (less than $40 \%$ ) in their circulating red blood cells ${ }^{2}$. On the other hand, homozygous individuals (HbSS) have two affected hemoglobin $\beta$ chains and develop sickle cell disease (SCD), which is characterized by a spectrum of conditions characterized by the sickling of red blood cells and vaso-occlusive events, with a significant impact on the morbidity and mortality of affected individuals ${ }^{3}$.

Traditionally, SCT has been seen as a benign condition, which does not require regular or specific medical treatment. However, recent reports have shown, over time, that important local and systemic complications may occur when these individuals are exposed to extreme situations. In these cases, sickling crises can be triggered even in individuals with SCT, especially when exposed to dehydration, low temperatures, infections, hypoxia, or after strenuous physical activities ${ }^{4,5}$.

Some serious complications have been associated with SCT, such as sudden death, exertional rhabdomyolysis, splenic stroke, hyposthenuria, renal papillary necrosis, and renal medullary carcinoma. Therefore, although rare, the possibility of an association between certain complications and SCT should not be excluded ${ }^{6}$.

Several manifestations in the maxillofacial complex have been associated with SCD, such as pallor of the oral mucosa, paresthesia of the mandibular nerve, delayed tooth eruption, hypomineralization of the enamel, and dentin, asymptomatic pulp necrosis (APN), gingival enlargement, mandibular osteomyelitis and excessive facial growth ${ }^{7,8}$. However, the same cannot be said concerning SCT, since there are no oral complications previously reported in this group of individuals.

APN is one of the possible oral complications in individuals diagnosed with SCD. This is due to the ability of sickle cells to adhere to the walls of the vascular endothelium, obstructing their lumen and generating a local vaso-occlusive phenomenon, which leads to hypoxia of the periapical region of the affected teeth, with subsequent $\mathrm{APN}^{9,10}$. There are no reports in the scientific literature describing the manifestation of APN in patients with SCT. However, taking into account the possibility of complications present in this group of individuals, when previously exposed to some special or extreme conditions, this relationship should not be excluded.

We report here a new clinical case of an extensive palatal swelling, arising from multiple APN, in a patient with SCT, thus discussing the possible relationship between APN and SCT.

\section{CASE REPORT}

A Brazilian female patient, 37-year-old, melanoderma, living in Switzerland, who was on vacation in Brazil, sought dental care at the Bahia Adventist Dental School (FADBA) with a complaint of "extensive swelling in the roof of the mouth", which had been progressively growing for the last four months. During the anamnesis, the patient reported having SCT, which never required medical treatment before. She also cited the death record of her younger brother due to complications of SCD. On intraoral physical examination, it was observed the presence of painful swelling in the anterior region of the hard palate on the left maxilla, extending from teeth 21 to 24 and measuring approximately three centimeters (Fig. 1).

Teeth associated with the lesion were clinically healthy, with no previously traumatic record. Also, an erosion on the palatal surface of anterior maxillary teeth was noted during the clinical examination, although the patient denying the consumption of acid substances or frequent vomiting episodes. A cone-beam computed tomography (CBCT) of the anterior maxilla was requested, and revealed the presence of a hypodense, unilocular lesion, extending from the periapical region of tooth 12 to tooth 24 , with the involvement of teeth

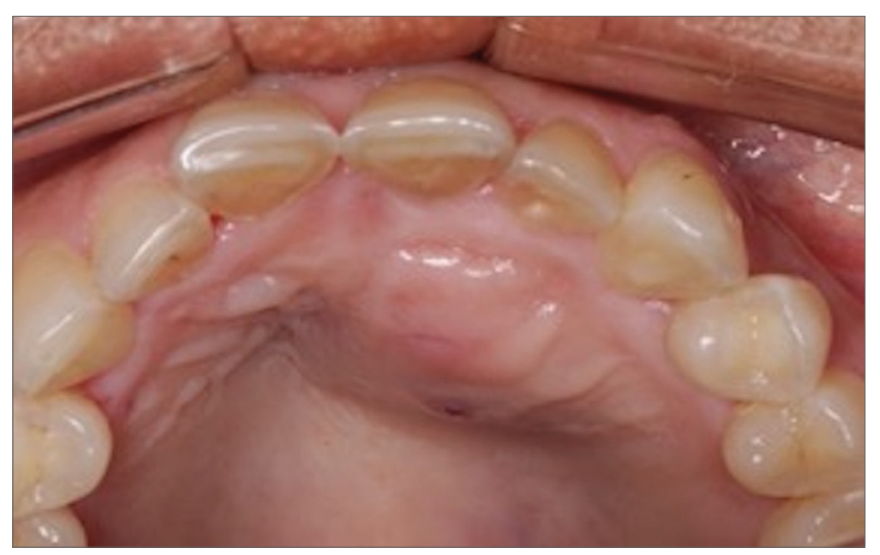

Figure 1. Palatal view of the extensive swelling involving patient's left maxilla. 
21, 22, 23 and the incisor canal (Fig. 2a). Its mesiodistal dimension was then measured in approximately 13.8 centimeters and revealed the fenestration of buccal cortical bone related to tooth 22 and 23 and extensive destruction of the palatal cortical bone (Fig. 2b). Also, it was observed osteolytic lesions involving the periapex of tooth 23 and 24 (Fig. $2 \mathrm{c}$ and 2 d).

Thermal (cold test) pulp vitality with tetrafluoroethane was performed on all superior teeth, which showed negative response only for teeth 23 and 24 . With the initial hypothesis of being a possible periapical lesion of endodontic origin due to APN and, based on tomographic and clinical findings, endodontic treatment of teeth 23 and 24 was indicated. Endodontic treatment was performed in a single session using a conventional rotary system under absolute isolation with root canal filling of teeth 23 and 24 with gutta-percha (Fig. 3b). During the opening of

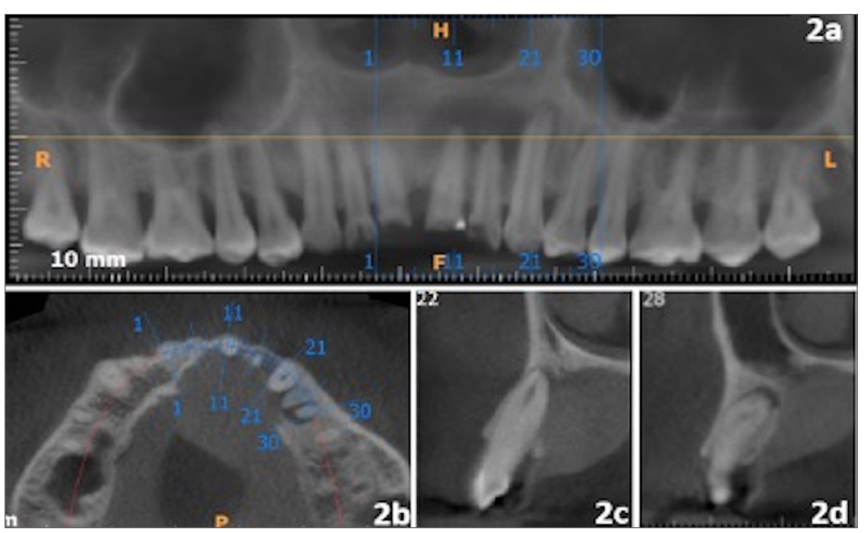

Figure 2. Panoramic reconstruction of $C B C T$ showing an extensive hypodense area in the left anterior maxilla (Fig. 2a); Axial section showing destruction of the palatal cortical bone (Fig. 2b); Parasagittal image showing the periapical involvement of teeth 23 (Fig. 2c) and 24 (Fig. 2d).

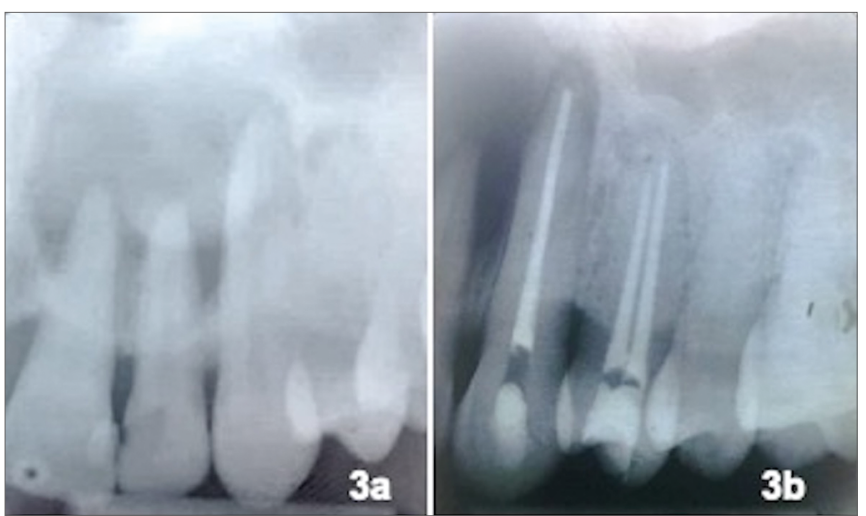

Figure 3. Periapical radiograph of tooth 23 and 24 after conventional root canal treatment (Fig. 3a); Visualization of the surgical wound and complete erosion of the palatal cortical bone (Fig. 3b); Closure of the surgical wound with resorbable sutures in position (Fig. 3c). the pulp chamber the endodontist found necrotic pulp tissue without pus in both teeth. A $2.5 \%$ sodium hypochlorite solution was used during and after the entire root canal instrumentation procedure. In addition, the patient was empirically treated with oral antibiotics (combination of amoxicillin $500 \mathrm{mg}$ three times a day and metronidazole $500 \mathrm{mg}$ three times a day) for seven days, in addition to analgesics and mouthwashes with $0.12 \%$ chlorhexidine digluconate.

Considering the patient's report of SCT and the family background of the severe manifestation of her brother's SCD, added to the unusual extension of the lesion and pulp necrosis without the presence of carious lesions or previous trauma to teeth 23 and 24 , an exploratory biopsy was indicated. A full-thickness palatine flap was made, which clinically confirmed the complete destruction of the palatal cortical bone (Fig. $4 \mathrm{a}$ and $4 \mathrm{~b})$. During the exploratory surgery, a friable tissue in the periapical region of the anterior maxillary teeth was found, that led the decision to perform the enucleation and curettage of the entire region.

The specimen collected during the exploratory biopsy and curettage of the lesion was sent for histopathological analysis, which confirmed the suspicion of periapical granuloma, possibly secondary to APN of teeth 23 and 24. In the histopathological analysis, the presence of Actinomyces sp was identified in the tissue samples collected (Fig. 5).

After a short stay in Brazil, which allowed only the patient's immediate post-surgical follow-up, she was instructed to seek an appointment with a hematologist to investigate other possible clinical manifestations of SCT. The patient remains asymptomatic and was instructed to follow her dental treatment with a local dentist in the country where she lives, which has been carried out for the last 24 months. Unfortunately, the patient's contact was lost, which made it impossible to perform new imaging tests to monitor the bone repair in the region, or to obtain information about the results of the new pulp vitality tests in the remaining teeth.
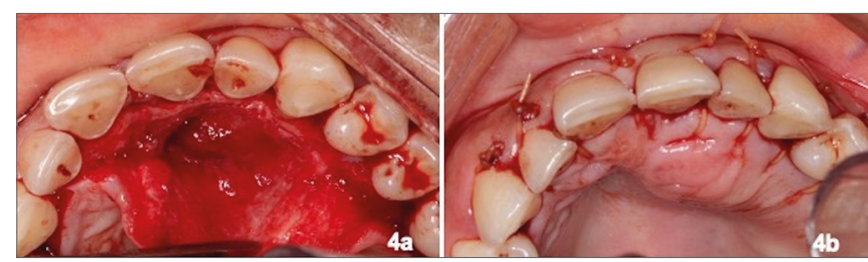

Figure 4. Visualization of the surgical wound and complete erosion of the palatal cortical bone (Fig. 4a); Closure of the surgical wound with resorbable sutures in position (Fig. 4b). 

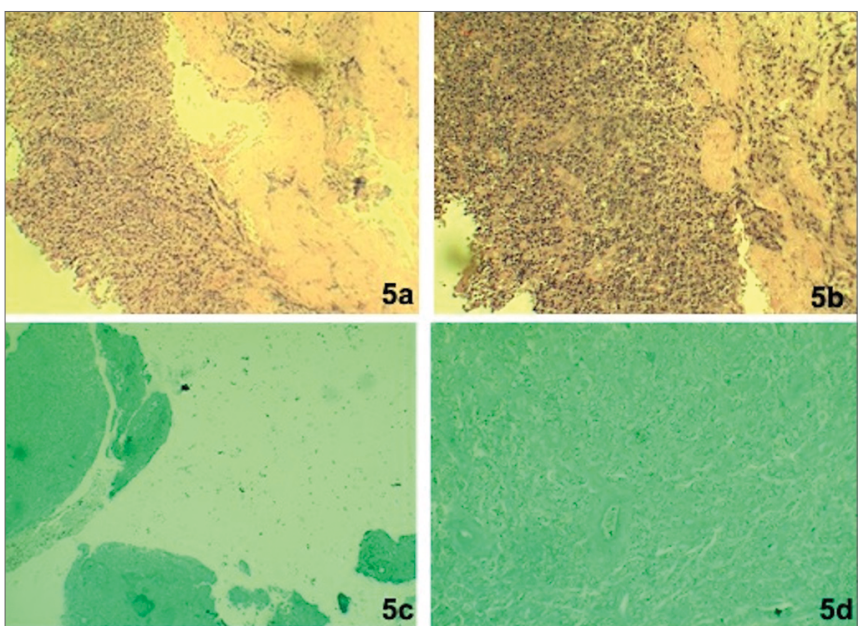

Figure 5. Histological examination of the periapical tissue removed. Granulation tissue associated with vascular proliferation and intense mixed inflammatory infiltrate (HE 50x and 100x, respectively) (Fig. 4a and Fig. 4b); Grocott's methenamine silver of periapical tissue, showing black staining round cysts from colonies of Actinomyces sp. (HE, 25x and 50x, respectively) (Fig. 4c and 4d).

\section{DISCUSSION}

For a complication to be attributed to SCT, it needs to occur more frequently in these individuals than in the general population. There is a range of complications already associated with SCT, which were classified by Tsaras, et al (2009) as: 1. defined; 2. probable; 3. possible; 4 . improbable or unproven ${ }^{11}$. These complications, mainly those classified as defined or probable, have already been reported in the scientific community through publications in numerous case series and literature reviews $\mathrm{s}^{2,6,12,13}$. On the other hand, there are published associations that have not yet been proven, or are improbable, such as avascular necrosis in the head of the femur, which corresponds to less than five cases being described in the literature ${ }^{14}$. Even with few published reports, these associations considered improbable should not be excluded from among the possible clinical manifestations of SCT due to their rarity. As far as we know, this is the first case report of APN in a patient with sickle cell trait.

APN is an oral manifestation proven to be associated with SCD patients, especially in adults ${ }^{15}$. However, there are no reports of maxillofacial manifestations of SCT, including APN. This event is characterized by vasoocclusion phenomena of the dental pulp microcirculation by sickle cells, especially during sickling crises, which can be triggered on account of extreme conditions, such as dehydration, metabolic acidosis, hypothermia, and strenuous physical exercises ${ }^{9,15}$. In this case report, we do not have information about a previous vaso-occlusive event from our patient, which made us hypothesize about the possible causes for this phenomenon to have occurred, which may have involved exposure to special conditions such as intense cold and dehydration in the last year of moving from Brazil to Switzerland.

The diagnosis of APN is still a real dilemma for clinicians and endodontists. This is because most clinical diagnoses are provided after routine radiographic findings that indicate periapical disease, or after infectious manifestations associated or not with toothache ${ }^{16,17}$. Unfortunately, the diagnosis for this condition is likely delayed in most cases, as clinicians may fail to consider that the dental pulp can become necrotic in the absence of caries, extensive periodontal disease, or previous trauma. In our case report, the patient was diagnosed with pulp necrosis only after the appearance of the extensive palatal swelling with painful symptoms was associated with drainage of purulent secretion, which guided the authors of this study to investigate the pulp vitality of teeth associated with the injury. The diagnostic dilemma related to this case reveals how complex the investigation of APN can be in asymptomatic patients with clinically healthy teeth and no history of trauma, especially in those more likely to have this issue, as in patients diagnosed within the spectrum of sickle cell disease.

Maxillary teeth may have reduced levels of oxygen saturation $(\mathrm{SpO} 2)$ when compared to mandibular teeth in individuals with $\mathrm{SCD}^{18}$. The authors of this present study hypothesize that the formation of microthrombi related to vaso-occlusive events may be more frequent in larger medullary spaces, such as in the maxilla. However, it is expected that, in areas such as the upper canines, higher $\mathrm{SpO} 2$ values will be found because they are surrounded by a bone of greater cortical density in the anterior maxilla ${ }^{18,19}$. In our clinical case, the teeth that responded negatively to the pulp vitality test (cold) and presented periapical hypodense lesions in the CBCT exam were the canine (tooth 23) and the first upper premolar (tooth 24), both on the patient's left maxilla. This does not exclude a likely occurrence of future pulp necrosis of the central and lateral upper incisors, which will have to be monitored clinically due to their close contact with the lesion.

An important issue for discussion is the fact that the necrotic teeth, despite being healthy, have periapical lesions that are secondarily infected by bacteria. This is a point of divergence in the scientific literature, as the aseptically necrotic pulp tissue is not capable of 
stimulating a periapical lesion in the absence of bacterial infection ${ }^{20}$. There is little information related to the investigation of the pathogenesis of periapical diseases in teeth that have suffered APN related to vaso-occlusive phenomena, especially in the identification of the presence of bacteria in the pulp tissue of those teeth. An analogy can be observed in studies that evaluated healthy teeth that suffered trauma and presented secondary infection, like in a study performed by Bruno et al. (2009), which investigated 20 necrotic teeth secondary to trauma with intact crowns and without periodontal disease through microscopic analysis. The authors found that $80 \%$ of the analyzed teeth had microorganisms in the necrotic pulp, justifying the development of a secondary odontogenic infection ${ }^{21}$.

Some of the hypotheses to explain the presence of these microorganisms in previously healthy tooth who suffered APN are: 1 . they are caused by bacteria of the oral cavity via gingival sulcus; 2 . by exposed dentinal tubules; 3. by cracks in enamel; or 4. they come from some distant site of infection in the body, reaching the pulp via a hematogenous route ${ }^{21.22}$. When bacteria find the necrotic pulp tissue, they multiply more easily, quickly developing a periapical infection ${ }^{23}$. Even though most studies that investigated bacterial colonization secondary to APN have occurred in teeth that have suffered trauma, they are still the basis for postulating the same mechanism in patients with SCD or SCT and who have suffered APN from specific vaso-occlusive phenomena. In the reported clinical case, it was possible to identify the presence of an extensive hypodense lesion in the left maxilla secondary to APN, involving the periapical region of canine and first ipsilateral upper premolar, secondarily infected by bacterial colonies of Actinomyces sp. As Actinomyces sp. are a group of bacteria commonly found in the gingival sulcus, the authors here postulate possible bacterial colonization by a periodontal route.

\section{CONCLUSION}

Although SCT is considered a benign condition in most affected individuals, scientific evidence suggests associations with rare important clinical manifestations in the face of extreme conditions involving hypoxia, hypothermia, acidosis, increased blood viscosity, dehydration and infections. These clinical manifestations in patients with SCT are relatively uncommon, but they should serve as a warning to health professionals, patients, and their families, who must be properly educated. This includes the clinical dentist and endodontists, who must be attentive to investigate the medical background of patients with reports of SCD in their family, or who already have the diagnosis of SCT, and who present APN without plausible explanation in healthy teeth. As far as we know, this is the first report of APN associated with an extensive periapical lesion in a patient with SCT. However, due to the rarity of this possible new clinical manifestation, more reports will be needed to prove a more consistent relationship between APN and SCT.

\section{REFERENCES}

1. Gibson JS, David CR. How benign is sickle cell trait? EBioMedicine. 2016 Sep;11:21-2.

2. Key NS, Vimal KD. Sickle-cell trait: novel clinical significance. Hematology Am Soc Hematol Educ Program. 2010;2010(1):418-22.

3. Bender MA. Sickle cell disease. GeneReviews [Internet]. 2003; [access in ANO Mês dia]. Available from: http://www.ncbi. nlm.nih.gov/books/NBK1377/

4. Goldsmith JC, Bonham VL, Joiner CH, Kato GJ, Noonan AS, Steinberg MH. Framing the research agenda for sickle cell trait: building on the current understanding of clinical events and their potential implications. Am J Hematol. 2012 Mar;87(3):340-6.

5. Jordan LB, Smith-Whitley K, Treadwell MJ, Telfair J, Grant AM, Ohene-Frempong K. Screening US college athletes for their sickle cell disease carrier status. Am J Prev Med. 2011 Dec;41(6):406-12.

6. Tantawy AAG. The scope of clinical morbidity in sickle cell trait. Egypt J Med Hum Genet. 2014;15(4):319-26.

7. Al-Jafar H, Dashti H, Al-Haddad SJ, Al-Gattan S, Al-Ramzi A. Dental alterations in sickle cell disease. J Dent Oral Care Med. 2016 Apr;2(2):203-7.

8. Javed F, Correa FOB, Almas K, Nooh N, Romanos GE, Al-Hezaimi K. Orofacial manifestations in patients with sickle cell disease. Am J Med Sci. 2013 Mar;345(3):234-7.

9. Kaya DA, Aktener BO, Unsal C. Pulpal necrosis with sickle cell anemia. Int Endod J. 2004 Sep;37(9):602-6.

10. Hebbel, RP, Greg MV, Karl AN. A systems biology consideration of the vasculopathy of sickle cell anemia: the need for multi-modality chemoprophylaxis. Cardiovasc Hematol Disord Drug Targets. 2009 Dec;9(4):271-92.

11. Tsaras G, Owusu-Ansah A, Boateng FO, Amoateng-Adjepong Y. Complications associated with sickle cell trait: a brief narrative review. Am J Med. 2009 May;122(6):507-12.

12. Fernando CHK, Mendis S, Upasena AP, Costa YJ, Williams HS, Moratuwagama D. Splenic syndrome in a young man at high altitude with undetected sickle cell trait. Patient Exp J. 2018 Jun;5(2):153-5.

13. Hu J, Nelson DA, Deuster PA, Marks ES, O'Connor FG, Kurina LM. Sickle cell trait and renal disease among African American US Army soldiers. Br J Haematol. 2019 May;185(3):532-40.

14. Sanders WJ. A rare case of avascular necrosis in sickle cell trait: a case report. BMC Hematol. 2018 Feb;18(1):1-4.

15. Costa CPS, Thomaz EBA, Souza SFC. Association between sickle cell anemia and pulp necrosis. J Endod. 2013 Feb;39(2):177-81. 
16. Bishop K, Briggs P, Kelleher M. Sickle cell disease: a diagnostic dilemma. Int Endod J. 1995 Nov;28(6):297-302.

17. Kelleher M, Bishop K, Briggs P. Oral complications associated with sickle cell anemia: a review and case report. Oral Surg Oral Med Oral Pathol Oral Radiol Endod. 1996 Aug;82(2):225-8.

18. Souza SFC, Thomaz EBAF, Costa CPS. Healthy dental pulp oxygen saturation rates in subjects with homozygous sickle cell anemia: a cross-sectional study nested in a cohort. J Endod. 2017 Dec;43(12):1997-2000.

19. D'Amico A. Functional occlusion of the natural teeth of man. J Prosthetic Dent. 1961;11(5):889-915.
20. Möller AJ, Fabricius L, Dahlén G, Ohman AE, Heyden G. Influence on periapical tissues of indigenous oral bacteria and necrotic pulp tissue in monkeys. Scand J Dent Res. 1981 Dec;89(6):475-84.

21. Bruno KF, Alencar AHG, Estrela C, Batista CA, Pimenta FC. Microbiological and microscopic analysis of the pulp of non-vital traumatized teeth with intact crowns. J Appl Oral Sci. 2009 Oct;17(5):508-14.

22. Taklan SA. A bacteriological study of the pulp of intact non-vital teeth. Int Endod J. 1974;7(2):75-7.

23. Yu C, Abbott PV. An overview of the dental pulp: its functions and responses to injury. Aust Dent J. 2007 Mar;52(Suppl 1):S4-S16. 